\title{
Las escorias de la central térmica GICC ELCOGAS como materia prima para la síntesis de materiales vitrocerámicos. Parte 2: Síntesis y caracterización de los materiales vitrocerámicos
}

\author{
M. AINETO ${ }^{1}$, A. ACOSTA' ${ }^{1}$ J.MA. RINCÓN² ${ }^{2}$ M. ROMERO ${ }^{2}$ \\ 'Universidad de Castilla La Mancha, Facultad de Ciencias Químicas, Departamento de Química Física, Área de Mineralogía Aplicada. \\ C/ Camilo José Cela s/n 13071 Ciudad Real \\ ${ }^{2}$ Grupo de Materiales Vítreos y Cerámicos, Instituto Eduardo Torroja de Ciencias de la Construcción, CSIC. C/Serrano Galvache 4, 28033 Madrid.
}

\begin{abstract}
Se expone en este trabajo la segunda parte de los resultados de la investigación sobre el comportamiento en fusión de las escorias y el proceso seguido para la obtención de materiales vitrocerámicos utilizando estas escorias como materia prima. A partir de una mezcla vitrificable basada en escorias, casco de vidrio y carbonato cálcico precipitado de azucarera, se ha obtenido por fusión a $1450^{\circ} \mathrm{C}$ un vidrio (ECSCP) que presenta un mecanismo de cristalización superficial.

Con el vidrio pulverizado se conformaron probetas cilíndricas de $40 \mathrm{~mm}$ de diámetro y $7 \mathrm{~mm}$ de altura aproximada, que fueron posteriormente sometidas a los tratamientos térmicos de desvitrificación a temperaturas entre 800 y $1100^{\circ} \mathrm{C}$, y tiempos de tratamiento entre 5 y 60 minutos, obteniéndose una serie de materiales vitrocerámicos de wollastonita-anortitagehlenita. Estos materiales han sido caracterizados y analizadas sus propiedades en función del tiempo y las temperaturas de desvitrificación.
\end{abstract}

Palabras clave: Residuos de gasificación de carbón, escorias GICC, desvitrificación, vitrocerámicos de wolastonita / anortita, materiales porosos.

The slag from ELCOGAS IGCC thermal power plant as raw material for the synthesis of glass-ceramic materials. Part 2: Synthesis and characterization of the glass-ceramic materials.

There are here reported the result of the second phase of the investigation on the melting behavior of the slag and the process followed to synthesize glass-ceramic materials using this slag as raw component. Starting from a vitrifying mixture based on slag, glass cullet and precipitated calcium carbonate coming from sugar refining, we have obtained the parent glass named ECSCP, which exhibit a surface tendency of crystallization.

Pressed specimens of $40 \mathrm{~mm}$ diameter and $7 \mathrm{~mm}$ height were conformed with the powdered ECSCP glass. The specimens were heat treated for crystalline phases development at temperatures between 800 and $1100^{\circ} \mathrm{C}$ during time intervals from 5 to 60 minutes.

A series of wollastonite-anorthite-gehlenite glass-ceramics has been synthesized of different characteristics depending on the time and temperature of devitrification.

Keywords: Coal gasification wastes, IGCC slag, devitrification, anorthite / wollastonite glassceramics, porous materials.

\section{INTRODUCCIÓN}

Los materiales vitrocerámicos son sólidos policristalinos obtenidos por la desvitrificación controlada de un vidrio. El vidrio original se sintetiza previamente con una composición de óxidos ajustada para dar lugar al desarrollo de fases cristalinas específicas. Las buenas propiedades de estos materiales se basan en su estructura, compuesta por una elevada concentración de microcristales, generalmente de un tamaño entre 10 y $1000 \mathrm{~nm}$, distribuidos uniformemente en una matriz vítrea (1). El aspecto básico a tener en cuenta para conseguir una buena microestructura es llevar a cabo la desvitrificación con un adecuado control del proceso para favorecer el desarrollo de un elevado número de cristales de pequeño tamaño.

La desvitrificación se produce cuando se somete al material vítreo a tratamientos térmicos que normalmente se dan en dos fases: una primera de nucleación, para promover la formación de gérmenes cristalinos estables, distribuidos de forma homogénea por todo el vidrio, y una segunda fase, a temperaturas más altas, de crecimiento de estos núcleos (2).

Otra de la vías de síntesis de materiales vitrocerámicos es la sinterización-cristalización de vidrio en polvo (3). El polvo prensado densifica a temperaturas relativamente bajas a través de un mecanismo de flujo viscoso; después se produce la cristalización mediante los tratamientos térmicos a temperatura y duración controlados para obtener la microestructura idónea. A veces, los dos fenómenos de densificación y cristalización se pueden dar en una sola etapa.

El proceso a seguir comienza con una primera etapa de fusión de la mezcla vitrificable, seguida de un enfriamiento brusco del fundido por fritado en agua para facilitar su fractura. El vidrio fritado es posteriormente molido hasta su pulverización y este vidrio en polvo es a continuación 
conformado a presión. A partir de la pieza conformada se efectúan los correspondientes tratamientos térmicos para la desvitrificación .

En los últimos años se ha investigado la obtención de materiales vitrocerámicos de construcción por reciclado de distintos tipos de residuos: de centrales térmicas convencionales (4), de incineradoras de residuos sólidos urbanos (5), de vidrios reciclados (6), y otros residuos industriales, pero no son conocidos estudios sobre aplicaciones vitrocerámicas de residuos procedentes de centrales térmicas GICC.

El objetivo de este trabajo es la obtención de materiales vitrocerámicos por desvitrificación de un vidrio sintetizado a partir de escorias GICC y otros materiales residuales. La obtención y caracterización del vidrio original ha sido descrita en un trabajo previo (7). La mezcla diseñada para obtener el vidrio de partida contiene un $40 \%$ de escorias GICC un 30\% de carbonato cálcico precipitado de azucarera y el 30\% restante, de casco de vidrio reciclado. Su composición está situada en torno al eutéctico a $1170^{\circ} \mathrm{C}$ del diagrama de fases $\mathrm{CaO}-\mathrm{Al}_{2} \mathrm{O}_{3}-$ $\mathrm{SiO}_{2}$. El vidrio original obtenido en la fusión de esta mezcla presenta un mecanismo de cristalización de tipo superficial, por lo que la obtención de los materiales vitrocerámicos se ha llevado a cabo por el procedimiento de sinterización y cristalización del vidrio en polvo conformado por prensado.

\section{PARTE EXPERIMENTAL}

\subsection{Preparación de muestras}

La fusión de la mezcla ECSCP se realizó en horno eléctrico a $1450^{\circ} \mathrm{C}$, manteniéndose a esa temperatura durante 1 hora. El fundido se enfrió colado sobre agua, dando lugar a una frita de color negro, que fue filtrada y secada en una estufa a $110^{\circ} \mathrm{C}$. La Tabla 1 muestra la composición química de esta frita determinada por FRX. La molienda del vidrio se llevó a cabo en un molino mecánico de ágata por tandas, separando cada 10 minutos las fracciones que quedaban por debajo de 160 micras. La granulometría final del polvo de vidrio ECSCP se midió por sedimentación en húmedo en un equipo Sedigraph 5100. El gráfico de este análisis granulométrico está representado en la Figura 1.

TABLA I. COMPOSICIÓN QUÍMICA (FRX) DEL VIDRIO ECSCP

\begin{tabular}{|l|c|c|c|c|c|c|c|c|c|c|}
\hline & $\mathrm{SiO}_{2}$ & $\mathrm{Al}_{2} \mathrm{O}_{3}$ & $\mathrm{Fe}_{2} \mathrm{O}_{3}$ & $\mathrm{MnO}$ & $\mathrm{MgO}$ & $\mathrm{CaO}$ & $\mathrm{Na}_{2} \mathrm{O}$ & $\mathrm{K}_{2} \mathrm{O}$ & $\mathrm{TiO}_{2}$ & $\mathrm{P}_{2} \mathrm{O}_{5}$ \\
\hline ECSCP & 53.14 & 14.40 & 3.17 & 0.06 & 1.63 & 20.56 & 5.00 & 1.40 & 0.43 & 0.40 \\
\hline
\end{tabular}

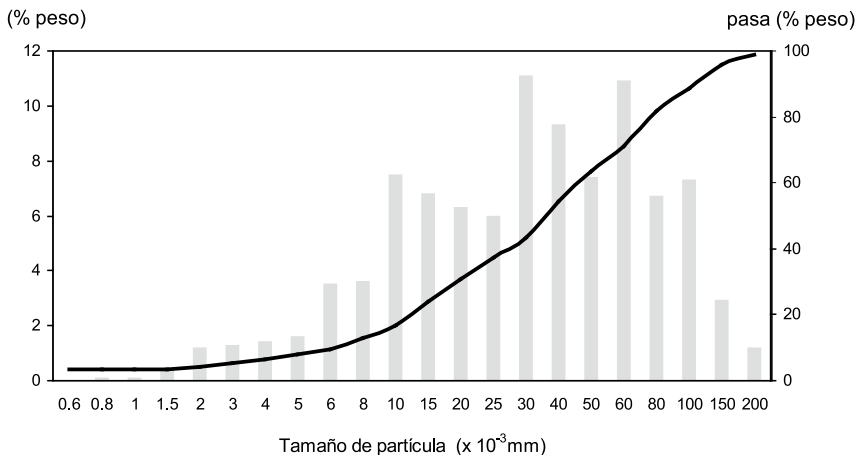

Fig. 1.- Análisis granulométrico e histograma del vidrio ECSCP pulverizado.
Con el vidrio pulverizado se prepararon probetas cilíndricas de $40 \mathrm{~mm}$ de diámetro y $7 \mathrm{~mm}$ de altura aproximada por prensado uniaxial a $20 \mathrm{MPa}$ durante 20 segundos. Cada probeta se preparó con $15 \mathrm{~g}$ de vidrio en polvo añadiendo un $3 \%$ de agua como aglomerante. Las muestras se secaron en estufa durante 24 horas y se reservaron en un desecador hasta el momento de ser introducidas en el horno para su desvitrificación.

\subsection{Tratamientos térmicos de desvitrificación}

Los tratamientos térmicos para la desvitrificación controlada del vidrio se llevaron a cabo a temperaturas de $800^{\circ}, 850^{\circ}, 900^{\circ}, 1000^{\circ}$ y $1100^{\circ} \mathrm{C}$, y tiempos de permanencia de 5, 10, 15, 30, 45, y 60 minutos. El proceso se llevó a cabo introduciendo toda la serie de probetas en el horno previamente calentado a la temperatura de ensayo prefijada. Una vez recuperada la temperatura se tomó ese momento como tiempo cero, y se fueron sacando las probetas en los intervalos de tiempo anteriormente señalados.

Las probetas utilizadas para los análisis por difracción de rayos- $X$, densidad y microestructura se dejaron enfriar al aire. Las demás se mantuvieron en un horno de recocido a $550^{\circ} \mathrm{C}$, durante al menos una hora, para luego dejar enfriar en su interior.

\subsection{Caracterización de los materiales vitrocerámicos}

La fases cristalinas desarrolladas se analizaron por difracción de rayos- $X$ (DRX) en un difractómetro PHILIPS PW1710 con rendija automática de divergencia y monocromador de grafito empleando la radiación Ka del cobre. El ensayo se realizó compactando en un porta de aluminio las muestras secadas a $110^{\circ} \mathrm{C}$ y pulverizadas a tamaños de partícula por debajo de 50 micras. Con los resultados de cristalización se estudió, para cada temperatura, la evolución en el tiempo de los picos de máxima intensidad de cada una de las tres fases detectadas.

El análisis microestructural se llevó a cabo por microscopía electrónica de barrido (MEB) sobre superficie de fractura. La observación de la porosidad se llevó a cabo en muestras sin atacar, mientras que los desarrollos cristalinos se observaron sobre fragmentos tratados con $\mathrm{HF}$ al $10 \%$ durante 60 segundos.

Las medidas de contracción y densidad se realizaron sobre las piezas tratadas durante 30 minutos a cada temperatura. La contracción o expansión de las piezas después de los tratamientos térmicos se midió como el porcentaje del incremento del diámetro respecto al diámetro inicial, considerando el valor medio de tres medidas efectuadas con un pie de rey.

Las medidas de densidad se realizaron en un picnómetro de Helio Accupyc y se llevaron a cabo sobre fragmentos en volumen y sobre material pulverizado hasta un tamaño de partícula menor de 50 micras.

\section{RESULTADOS Y DISCUSIÓN}

Los análisis de difracción de rayos- $X$ han mostrado que la desvitrificación a tiempos cortos es posible a partir de $900^{\circ} \mathrm{C}$, siendo wollastonita $\left(\mathrm{CaSiO}_{3}\right)$, anortita $\left(\mathrm{CaAl}_{2} \mathrm{Si}_{2} \mathrm{O}_{8}\right)$ y gehlenita $\left(\mathrm{Ca}_{2} \mathrm{Al}_{2} \mathrm{SiO}_{7}\right)$ las fases cristalinas desarrolladas, tal y como se recoge en la Tabla 2. 

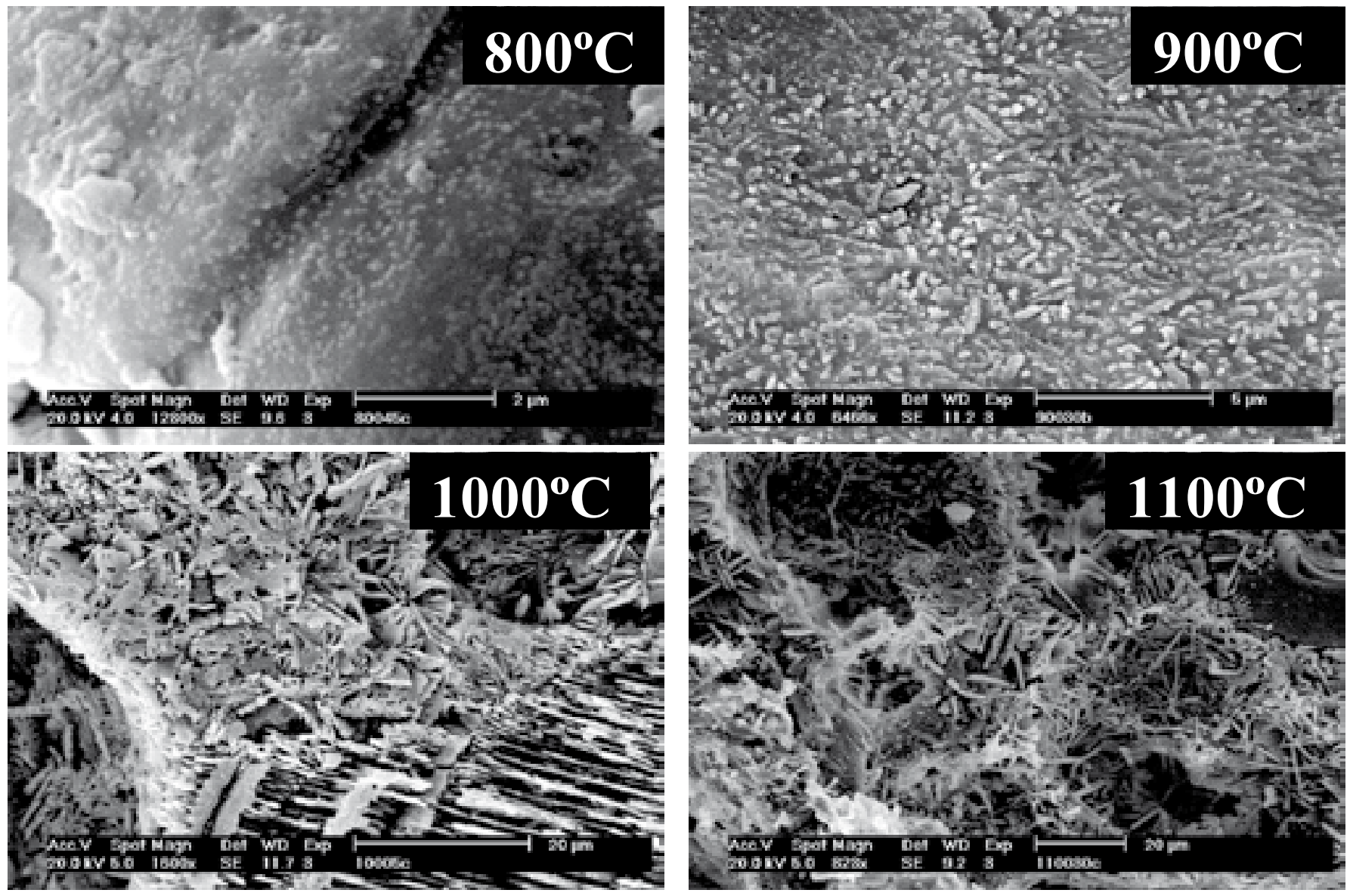

Fig. 2.- Imágenes por microscopía electrónica de barrido sobre superficies de fractura de probetas tratadas durante 45 minutos a $800^{\circ} \mathrm{C}, 30$ minutos a $900^{\circ} \mathrm{C}, 5$ minutos a $1000^{\circ} \mathrm{C}$ y 30 minutos a $1100^{\circ} \mathrm{C}$.

TABLA II. FASES CRISTALINAS DETECTADAS POR DRX EN FUNCIÓN DEL TIEMPO Y LA TEMPERATURA DE TRATAMIENTO TÉRMICO. ENTRE PARÉNTESIS LAS FASES QUE COMIENZAN A CRISTALIZAR: AMF: AMORFO; A: ANORTITA; W: WOLASTONITA; G: GEHLENITA; O: ORTOCLASA.

\begin{tabular}{|c|c|c|c|c|c|}
\hline \multirow{2}{*}{$\begin{array}{c}\text { Tiempo } \\
(\mathrm{min})\end{array}$} & \multicolumn{5}{|c|}{ Temperatura de tratamiento $\left({ }^{\circ} \mathrm{C}\right)$} \\
\cline { 2 - 6 } & 800 & 850 & 900 & 1000 & 1100 \\
\hline 5 & Amf & Amf & W, A & W, A & W,A,(G) \\
\hline 10 & Amf & Amf & W, A & W,A, (G) & W,A, (G) \\
\hline 15 & Amf & W & W,A, (G) & W,A,G & W, A \\
\hline 30 & Amf & W, A, (G) & W,A,G & W,A,G & W, A, (O) \\
\hline 45 & Amf & W,A,G & W,A,G & W,A,G & W, A, (O) \\
\hline 60 & Amf & W,A,G & W,A,G & W,A,G & W, A, (O) \\
\hline
\end{tabular}

La Figura 2 muestra la microestructura observada por MEB en muestras seleccionadas. A $800^{\circ} \mathrm{C}$ se obtiene un sinterizado homogéneo de color negro. A esta temperatura no se detecta por DRX crecimiento cristalino en los primeros sesenta minutos de tratamiento, si bien, en un ensayo durante 24 horas se observan los picos correspondientes al desarrollo de las tres fases cristalinas anteriormente mencionadas. No obstante, la imagen de microscopía electrónica de barrido (Fig. 2) correspondiente a un tratamiento a $800^{\circ} \mathrm{C}$ durante 45 minutos, sí permite apreciar algunos dominios con pequeños núcleos cristalinos de tamaño submicrónico.

Los materiales vitrocerámicos obtenidos a $850^{\circ} \mathrm{C}$ presentan un mismo aspecto externo con color negro o gris oscuro. La primera fase que aparece es wollastonita, que comienza a detectarse por difracción de rayos- $X$ a partir de 15 minutos de tratamiento. A tiempos más prolongados aparecen también las restantes fases. Las imágenes observadas por MEB en las probetas tratadas durante 30 minutos muestran alta proporción de fase vítrea, que se ve reducida a tiempos más prolongados.

Los tratamientos a $900^{\circ} \mathrm{C}$ dan lugar a materiales sinterizados de color negro en el interior y pardo-amarillento en su superficie externa. El crecimiento cristalino se produce con gran rapidez, obteniéndose por difracción de rayos- $X$ valores altos de intensidad de picos desde tiempos de 5 minutos. El desarrollo cristalino a esta temperatura (Fig. 2) consiste en cristales aislados de forma alargada y tamaño medio de 1 micra, inmersos en la fase vítrea.

Las probetas tratadas a $1000^{\circ} \mathrm{C}$ presentan color pardo oscuro con pequeños puntos superficiales de color amarillo, que ocupan una mayor superficie a medida que aumenta el tiempo de permanencia a esta temperatura. En el centro de la pieza se produce un abultamiento o deformación por formación de burbujas y su interior es negro. La desvitrificación es prácticamente completa, y se observan diferentes hábitos de crecimiento cristalino, en forma de agujas o plaquetas que se disponen en estructuras laminares o tridimensionales (Fig. 2).

A $1100^{\circ} \mathrm{C}$ los cambios en el aspecto externo son más notables, con coloración gris claro a tiempos cortos. A partir de 15 minutos, el tratamiento da lugar a materiales cuyo color predominante es el amarillo, quedando en el interior de la probeta algunas zonas más oscuras. En este caso se advierten 


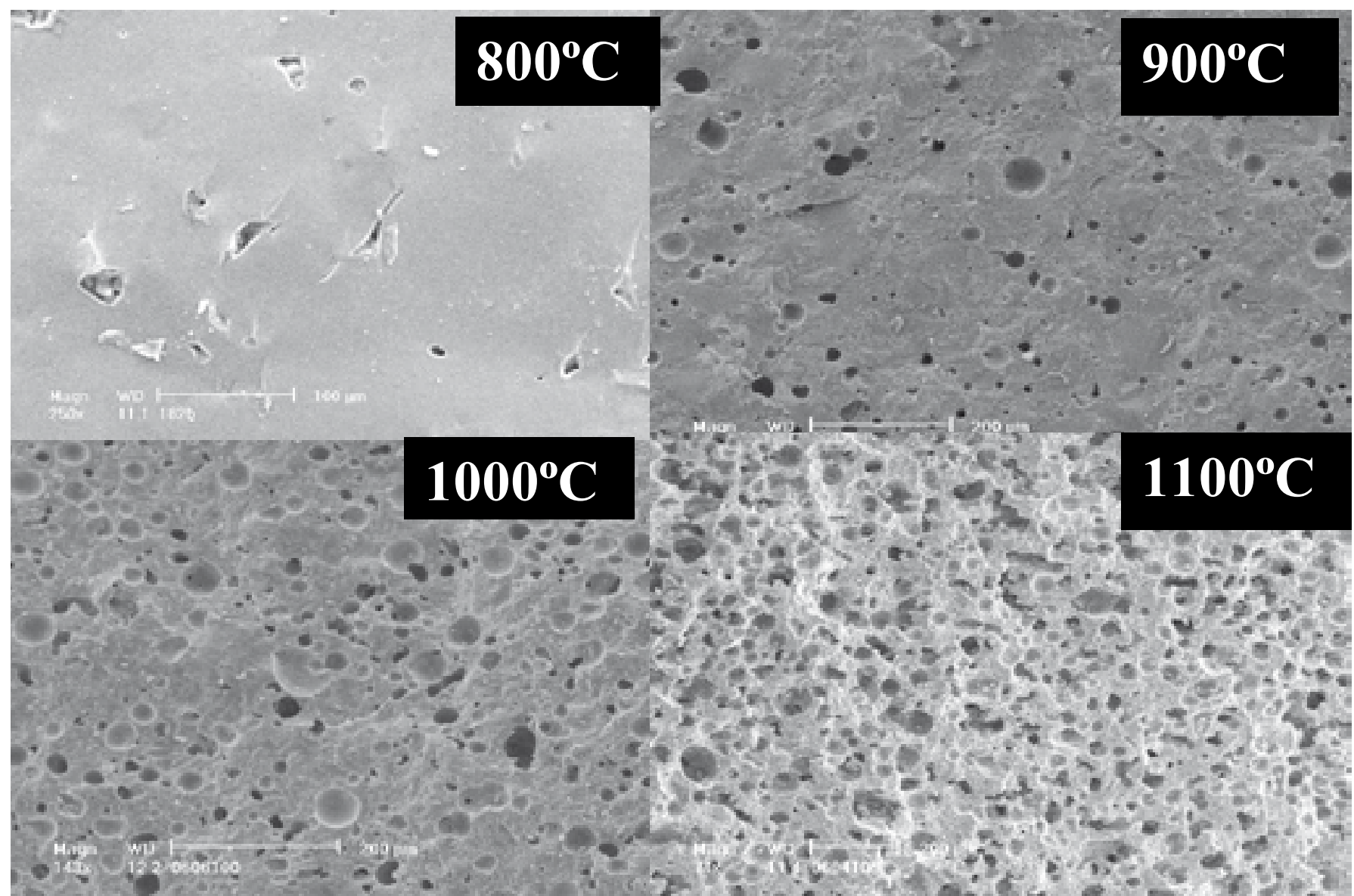

Fig. 3.- Imágenes de microscopía electrónica de barrido de los materiales tratados 30 minutos a $800^{\circ}, 900^{\circ}, 1000^{\circ}$ y $1100^{\circ} \mathrm{C}$.

también deformaciones superficiales como consecuencia de la aparición de burbujas. De igual forma, a $1100^{\circ} \mathrm{C}$ las fases cristalinas sufren algunos cambios como la disolución total de la gehlenita a partir de 10 minutos de tratamiento, junto a la aparición de algunos picos correspondientes a una nueva fase que puede tratarse de una ortoclasa. La microestructura desarrollada a esta temperatura es similar a la observada a $1000^{\circ} \mathrm{C}$ (Fig. 2).

El cambio de coloración observado en las piezas que se han obtenido a diferentes temperaturas se puede atribuir a cambios en el estado de oxidación del hierro.

La Figura 3 muestra la evolución de la porosidad tras 30 minutos de tratamiento térmico a diferentes temperaturas. A $800^{\circ} \mathrm{C}$ se obtiene un material denso que va evolucionando con la temperatura hacia la formación de poros aislados a partir de $\operatorname{los} 900^{\circ} \mathrm{C}$. Esta porosidad cerrada sigue creciendo a $1000^{\circ} \mathrm{C}$ hasta transformarse a $1100^{\circ} \mathrm{C}$ en una estructura porosa interconectada similar a la de las espumas cerámicas(8), con poros distribuidos de forma homogénea y con tamaños desde 2-3 $\mu \mathrm{m}$ hasta $100 \mu \mathrm{m}$ según los tiempos de tratamiento.

Como consecuencia de los procesos de densificación y de desarrollo de porosidad las piezas experimentan cambios dimensionales que se traducen en una contracción máxima del $20 \%$ a $800^{\circ} \mathrm{C}$ disminuyendo progresivamente hasta llegar a una ligera expansión y deformación superficial a $1100^{\circ} \mathrm{C}$ motivada por la liberación de burbujas gaseosas.

El análisis de densidad en picnómetro de helio sobre muestras tratadas durante 30 minutos a las diferentes temperaturas de tratamiento térmico se muestra en la Figura 4. Las muestras se examinaron en polvo de tamaño de partícula

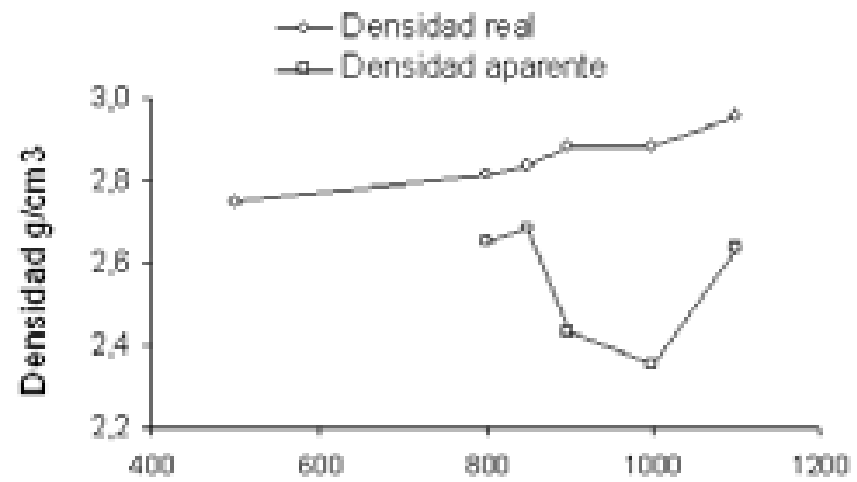

Temperatura de desvitrificación ${ }^{\circ} \mathrm{C}$

Fig. 4.- Variación de la densidad en función de la temperatura de desvitrificación.

menor de 50 micras para obtener los datos de densidad real, $\mathrm{y}$ en fragmentos de $0,25 \mathrm{~g}$ de peso aproximadamente, para el cálculo de la densidad aparente. El gráfico comparativo de ambas medidas de densidad muestra un incremento de la densidad real (muestra en polvo) a medida que aumenta la temperatura de tratamiento, como consecuencia de la disminución de la proporción de fase vítrea en el material vitrocerámico. En cuanto a la densidad aparente (muestra en volumen), la muestras dan valores más bajos al aumentar la temperatura hasta $1000^{\circ} \mathrm{C}$, como consecuencia del incremento de la porosidad cerrada. El material vitrocerámico 
obtenido a $1100^{\circ} \mathrm{C}$, que presenta un mayor desarrollo poroso, experimenta un aumento en su valor de densidad debido a la intercomunicación y apertura de los poros.

\section{CONCLUSIONES}

Se ha demostrado la viabilidad de la utilización de las escorias GICC en la producción de materiales vitrocerámicos. Como resultado de esta investigación, han sido sintetizados una serie de materiales en los que cristalizan wollastonita $\left(\mathrm{CaSiO}_{3}\right)$ y anortita $\left(\mathrm{CaAl}_{2} \mathrm{Si}_{2} \mathrm{O}_{8}\right)$ como fases mayoritarias y gehlenita $\left(\mathrm{Ca}_{2} \mathrm{Al}_{2} \mathrm{SiO}_{7}\right)$ como fase minoritaria. Estos materiales vitrocerámicos presentan distintas características en función de la temperatura y tiempo de duración de los tratamientos térmicos, obteniéndose materiales densos policristalinos a $900^{\circ} \mathrm{C}$. Los tratamientos a temperaturas más altas han promovido un desarrollo poroso paralelo a la desvitrificación, que evoluciona en función de las variables temperatura/ tiempo desde una porosidad cerrada a $1000^{\circ} \mathrm{C}$ hasta porosidad abierta al incrementarse la temperatura dando lugar a $1100^{\circ} \mathrm{C}$ a materiales vitrocristalinos porosos similares a las espumas cerámicas. Este desarrollo poroso es provocado por la existencia de gases que permanecían disueltos en el vidrio original y que son liberados cuando se alcanza la temperatura de reblandecimiento del material.

\section{AGRADECIMIENTOS}

Las siguientes entidades han colaborado en la financiación de esta investigación: la Comisión Interministerial de Ciencia y Tecnología a través del proyecto REN2001-0912/TECNO; las empresas ELCOGAS S.A y Azucarera EBRO S.L.

\section{BIBLIOGRAFÍA}

1. J.M. Fernández Navarro, "El estado vítreo y la estructura de los vidrios" pp. 47-123, en El Vidrio, Consejo Superior de Investigaciones Científicas, Fundación Centro Nacional del Vidrio. Madrid (España) 1991.

2. R.D.Rawlings, en "Glass-Ceramic Materials: Fundamentals and Applications", Mucchi Editore, Modena, (Italia) 1997.

3. I. Gutzow, R. Pascova, A. Karamanov, J. Schmelzer, “The kinetics of surface induced sinter crystallisation of glass powders". J. Mater. Sci., 33, 5265-5273 (1998).

4. A.A. Francis, R.D. Rawlings, R. Sweeney, A.R. Boccaccini, "Processing of coal ash into glass ceramic products by powder technology and sintering" Glass Technology, 43, (2), 58-62 (2002).

5. M. Romero, R.D. Rawlings, J.M. Rincón, "Crystal nucleation and growth in glasses from inorganic wastes of urban incineration" J. Non-Cryst. Solids, 271 (1-2), 106-118 (2000).

6. J. Martín-Márquez, M. Romero, J.Ma. Rincón, “Efecto de un fluidificante vítreo en la sinterización de una arcilla para gres porcelánico" en La investigación de arcillas en geología, agricultura, medio ambiente y ciencia de materiales, 247-250 (2002).

7. M. Aineto, A. Acosta: “Las escorias de la central térmica GICC ELCOGAS como materia prima para la síntesis de materiales vitrocerámicos. Parte 1: Comportamiento en fusión de las escorias GICC y obtención del vidrio original", Bol. Soc. Esp. Ceram. V. 44,6,399-404 (2005)

8. P. Colombo, “Ceramic foams. Fabrication, properties and applications" Key Eng. Mater. 206-213 1913-1918 (2002).

Recibido: 10.01 .05

Aceptado: 17.06 .05 